\title{
Comparative feeding habits of polychelid lobsters in the Western Mediterranean deep-sea communities
}

\author{
J. E. Cartes, P. Abelló \\ Institut de Ciències del Mar, Passeig Nacional s/n, E-08039 Barcelona, Spain
}

\begin{abstract}
Feeding habits of the 2 deep-water species of polychelid lobsters occurring in the Balearic Basin (Western Mediterranean) were examined. Samples were taken with an OTSB-14 bottom trawl from depths between 550 and $2300 \mathrm{~m}$ in 1988-89. Polycheles typhlops and Stereomastis sculpta occupy a well-defined trophic niche, between the uppermost level of the bottom sediment and the water layer immediately adjacent to the sea bed. Scavenging plays an important role in feeding. However, ontogenetic changes in the diet were clear in both species. Small individuals based their diet on epibenthic peracarid crustaceans, whereas scavenging and ingestion of inert remains became more important in the adult diet. The trophic habits of the 2 species were rather similar; differences recorded are more consistent with depth-related changes in available prey resources than with changes in the position of the 2 species in the food web. The progressive decrease of abundance of $P$. typhlops with increasing depth coincided with greatly reduced densities of some of its preferential prey (i.e. Calocaris macandreae, Cirolana borealis or euphausiids) and with a different feeding strategy. S. sculpta shows a more microphagic and passive feeding habit. Feeding activity, quite low in both species, was lower in $S$. sculpta. All these results suggest that $S$. sculpta is better adapted to the more oligotrophic conditions found on the lower slope. Competitive trophic interaction may also account for the separate depth distributions of the 2 species. These interactions may be especially important on the deep-sea bottoms in the Mediterranean Sea, where environmental parameters like temperature and salinity are constant.
\end{abstract}

\section{INTRODUCTION}

The Polychelidae (Decapoda, Palinuroidea) are one of the most characteristic families in deep-sea communities, with different species distributed in bathyal and abyssal oceanic regions (Kensley 1968, Wenner \& Boesch 1979, Gore 1984, Saint Laurent 1985, Abelló \& Valladares 1988). Polycheles typhlops Heller, 1862 and Stereomastis sculpta (S.I. Smith, 1880) are two of the most characteristic and abundant species in bathyal mud assemblages in the Western Mediterranean (Cartes 1991, Cartes et al. in press). P. typhlops is one of the dominant species in the middle subzone (500 to $1200 \mathrm{~m}$ ) of the continental slope (Abelló \& Valladares 1988, Abelló et al. 1988, Cartes 1991). S. sculpta is one of the more abundant species in the lower subzone (below 1200 m) (Pérès 1985, Cartes 1991, Cartes et al. in press). The separation in the depth ranges of these 2 species is quite clear. Their distribution ranges do overlap below $1000 \mathrm{~m}$, but densities of $P$. typhlops are very low there (Abelló \& Cartes in press). The size ranges of these species are similar. Environmental conditions of the Mediterranean deep-water mass in the bathyal zone are tairly constant: temperature $12.7^{\circ} \mathrm{C}$ and salinity 38.4 (Fredj \& Laubier 1985, Hopkins 1985).

Some isolated data have been published on the trophic habits of various polychelidae of the genus Stereomastis by Wenner (1979) and of the abyssal genus Willemoesia by Gore (1984) in the Atlantic. The feeding pattern of Polycheles typhlops has occasionally been studied. Santucci (1932) reported that feeding in this species was chiefly based on scavenging. In contrast, Lagardère (1977) described this species as a predator of bathypelagic crustaceans, with scavenging activity merely a complement to the diet. Based on the analysis of a few specimens, this same author suggested that the feeding pattern of S. sculpta was similar to that of $P$. typhlops. Some preliminary data on the feeding habits of P. typhlops and S. sculpta were presented by Cartes \& Abelló (1990). 
The object of the present study was to describe the diets of these 2 species over their entire distribution ranges and to analyze the effect of depth on diet. Given the similar morphology and size of the 2 species, types of prey could be similar although the precise species preyed upon may vary as a function of the differential distribution ranges of the species. Consideration has also been given to the level of dietary similarity as a possible contributing factor to the distinctly separate depth distributions of these species and to the ecological role of this crustacean group, which is characteristic in, and well adapted to, the deep-sea environment.

\section{MATERIALS AND METHODS}

Specimens were collected from the Balearic Basin using an otter trawi semi-balioon (OTSB-14) (Merret \& Marshall 1981) in July and October 1988 (1000 to $2300 \mathrm{~m} ; 15$ samples) and July and October 1989 (550 to $750 \mathrm{~m}$; 9 samples). A total of 459 specimens of Polycheles typhlops and 1271 specimens of Stereomastis sculpta were dissected. The stomach fullness $(f)$ of each specimen was recorded; foreguts for which $f<20 \%$ were classified as empty, while those for which $f \geq 50 \%$ were classified as full. Food-containing stomachs were preserved in $70 \%$ alcohol. Individual size (cephalothorax length, CL) was also recorded.

In all, the contents of 193 Polycheles typhlops stomachs and 163 Stereomastis sculpta stomachs were studied. The diet composition was analyzed by depth intervals and in the case of $P$. typhlops from the upper middle slope also by season (see Table 1). The diet was also analyzed by comparing size classes of the same species. Three size classes were considered: $<20 \mathrm{~mm}, 20-30 \mathrm{~mm}_{1}>30 \mathrm{~mm} \mathrm{CL}$ in P. typhlops; $<16$ $\mathrm{mm}, 16-25 \mathrm{~mm},>25 \mathrm{~mm}$ CL in S. sculpta. Specimens in the smallest size class were immature; those in the 2 largest size classes were mature (Abelló \& Cartes in press).

Diets are presented in terms of percentage frequency of occurrence $(\% \mathrm{~F})$ and percentage abundance (\%N) by prey item (Hyslop 1980, Cartes \& Sardà 1989). Quantification was relatively simple (Cartes 1991). The mandibles of Polychelidae contain a well-developed cutting incisor process but no molar process. Thus, prey items are usually found whole or in a few, readily identifiable fragments. Foraminiferans were not quantified in this study and only the percentage occurrence of these organisms was recorded. Percentage volume values for the different prey groups have been expressed by the subjective points method (Swynnerton \& Worthington 1940). Prey identification was carried out to species level whenever possible.
To detect possible changes in the daily feeding pattern of Polycheles typhlops a continuous 24 h sampling was performed on the upper middle slope. Samples of both species collected from below $1000 \mathrm{~m}$ at different times of the day were analyzed. The occurrence of significant differences in foregut fullness was tested using a homogeneity chi-squared test. A subjective estimate of the state of digestion of prey was also established (Amezaga 1988, Sardà \& Valladares 1990, Cartes 1991).

Cumulative trophic diversity curves (Mauchline \& Gordon 1985) for both species were constructed using the Shannon-Wiener index (Shannon \& Weaver 1963). Asymptotic stabilization of the curves yielded the minimum number of stomachs that need to be analyzed to produce sufficiently reliable results.

Multivariate cluster analysis was used to analyze general affinities between the samples (diets) of the 2 species. Each sample combined the individuals taken in one haul. Uncommon prey items that ocurred only once in the samples were removed from the data matrix. Linear correlation (with previous logarithmic transformation of the data) was used as the similarity index. The aggregation algorithm used was the Unweighted Paired Method using Arithmetic Averages (UPGMA).

For purposes of comparison samples were aggregated according to species, depth strata and season. Overlap between the diets was calculated using the Schoener (1974) index and trophic diversity was established using the Shannon-Wiener index. These 2 indexes were calculated for each of the resulting sample groups established.

\section{RESULTS}

The basic prey type for the 2 species studied consisted of fish remains and benthic peracarid crustaceans. Cumulative trophic diversity values stabilized for both species after analysis of the contents of 15 to 20 stomachs. There is a low degree of prey manipulation; the ability to ingest prey items whole has been confirmed on different occasions.

\section{Sample aggregations}

Linear correlation analysis pointed to a clear separation between the samples of the 2 polychelid species (Fig. 1). Only one Polycheles typhlops sample (from the lower middle slope) was associated with Stereomastis sculpta. No evident groups were detected within either of the 2 main clusters discriminated by the analysis (Fig. 1). 


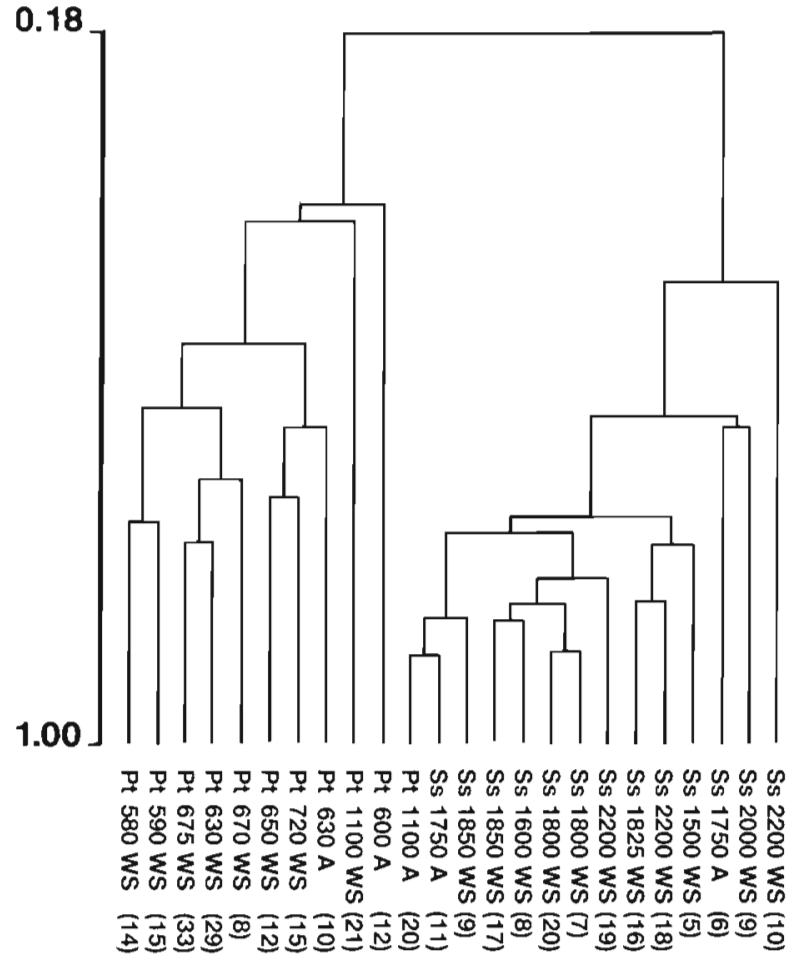

Fig. 1. Polycheles typhlops (Pt) and Stereomastis sculpta (Ss). Aggregation of foregut content samples for 2 polychelid species (linear correlation-UPGMA). The number of foregut contents studied in each sample is given in brackets, with the mean depth and time of year. WS: samples taken between winter and summer; A: samples taken in autumn

\section{Diet composition}

Polycheles typhlops: upper middle slope (550 to $750 \mathrm{~m}$ ). In spring and summer samples the diet was based on fish remains, the burrowing macruran Calocaris macandreae, and remains of the shrimp Aristeus antennatus. Other important prey were the mysid Boreomysis arctica and the isopod Cirolana borealis. These prey together accounted for nearly $60 \%$ of the diet (Table 1).

In autumn the most important prey were fish remains and Calocaris macandreae. Together with echinoids, euphausiids (Meganyctiphanes norvegica) and gammarid amphipods (Lysianassidae), these prey items accounted for around $60 \%$ of the diet. The share of euphausiids in the diet was quite high (Table 1, Fig. 2).

Polycheles typhlops: lower middle slope (1000 to $1200 \mathrm{~m})$. In terms of abundance (\%N) fish remains, asellid isopods (Munnopsurus atlanticus), pteropod remains, and polychaetes (Aphroditidae) contributed about $60 \%$ of the diet (Table 1). Results in terms of frequency of occurrence were similar, and foraminiferans were also common.
In terms of percentage volume, the diet appeared considerably more diverse than in the samples from the upper middle slope (Fig. 2).

Polycheles typhlops: lower slope $(1400$ to $1900 \mathrm{~m})$. Twenty-five individuals, all immature ( $\mathrm{CL}: 9$ to $19 \mathrm{~mm}$ ), of $P$. typhlops were analysed. Only 7 of the stomachs contained food remains. The prey were asellid isopods [Iyarachna sp. (2), Munnopsurus atlanticus (2)], an unidentified isopod (1), gammarid amphipods [Rhachotropis sp. (1), Pardaliscidae (1)], pteropod debris with the encrusting foraminiferan Ammolagena sp. (2), aphroditid polychaetes (2), and fish scales (1). These results suggest a diet based on suprabenthic crustaceans.

Stereomastis sculpta: lower slope (1400 to $2300 \mathrm{~m})$. Diet analysis was performed separately for the samples taken above and below $2000 \mathrm{~m}$ (Table 1), since this depth constitutes an ecological discontinuity in deca-

UPPER MIDOLE SLOPE |WS|

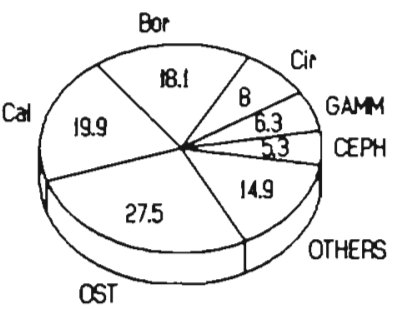

UPPER MIDOLE SLOPE |A|

Fig. 2. Polycheles typhlops Proportion (by volume) of prey species by group in the diet by depth interval and season. POL: polychaetes; NAT: natantian decapods; Pont: Pontophilus norvegicus; Cal: Calocaris macandreae; EUPH: euphausiids; Bor: Boreomysis arctica; GAMM gammarid amphipods; Cir: Cirolana borealis; ASELL: asellid isopods; ECH: echinoids; CEPH: cephalopods; OST: fishes

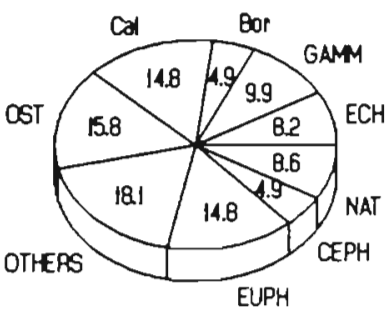

LOWER MIDOLE SLOPE

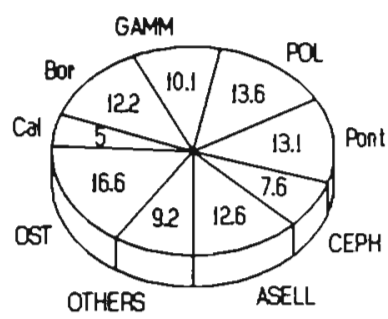


Table 1 Polycheles typhlops and Stereomastis sculpta. Diet composition of the 2 polychelid species. \%F: percentage frequency of occurrence; \%N: percentage abundance of prey item

\begin{tabular}{|c|c|c|c|c|c|c|c|c|c|c|}
\hline \multirow{5}{*}{ No. of specimens: } & \multicolumn{6}{|c|}{ Polycheles typhlops } & \multicolumn{4}{|c|}{ Stereomastis sculpta } \\
\hline & \multicolumn{4}{|c|}{$550-750 \mathrm{~m}$} & \multirow{3}{*}{\multicolumn{2}{|c|}{$\begin{array}{c}1000-1200 \mathrm{~m} \\
\text { Jul-Oct } \\
41\end{array}$}} & \multirow{3}{*}{\multicolumn{2}{|c|}{$\begin{array}{c}1400-1900 \mathrm{~m} \\
\mathrm{Jul}-\mathrm{Oct} \\
99\end{array}$}} & \multirow{3}{*}{\multicolumn{2}{|c|}{$\begin{array}{c}2000-2300 \mathrm{~m} \\
\text { Jul } \\
56\end{array}$}} \\
\hline & \multirow{2}{*}{\multicolumn{2}{|c|}{$\begin{array}{c}\text { Mar-Jul } \\
104\end{array}$}} & \multirow{2}{*}{\multicolumn{2}{|c|}{$\begin{array}{l}\text { Oct } \\
22\end{array}$}} & & & & & & \\
\hline & & & & & & & & & & \\
\hline & $\% \mathrm{~F}$ & $\% \mathrm{~N}$ & $\% \mathrm{~F}$ & $\% \mathrm{~N}$ & $\% \mathrm{~F}$ & $\% \mathrm{~N}$ & $\% \mathrm{~F}$ & $\% \mathrm{~N}$ & $\% \mathrm{~F}$ & $\% N$ \\
\hline \multicolumn{11}{|l|}{ HYDROZOA } \\
\hline Stephanoscyphus spp. & - & - & - & - & 2.4 & 1.8 & - & - & - & - \\
\hline POLYCHAETA & 3.9 & 1.8 & 4.5 & 2.7 & 12.2 & 10.9 & 12.1 & 6.7 & 14.3 & 8.4 \\
\hline Aphroditida & 1.0 & 0.4 & - & - & 12.2 & 9.1 & 10.1 & 5.2 & 7.1 & 3.4 \\
\hline Glycera sp. & - & - & - & -- & - & - & 1.0 & 0.5 & - & - \\
\hline Nephthys sp. & 1.0 & 0.4 & 4.5 & 2.7 & - & - & - & - & 1.8 & 0.8 \\
\hline Unidentified & 1.9 & 0.9 & - & - & 2.4 & 1.8 & 2.0 & 1.0 & 5.4 & 2.5 \\
\hline NEMATODA & - & - & - & - & - & - & 1.0 & 0.5 & 3.6 & 1.7 \\
\hline \multicolumn{11}{|l|}{ CRUSTACEA } \\
\hline \multicolumn{11}{|l|}{ Decapoda natantia } \\
\hline Bathypelagic & 3.8 & 1.8 & - & - & - & - & 1.0 & 0.5 & 3.6 & 3.6 \\
\hline Genadas elegans & - & - & - & - & - & - & 1.0 & 0.5 & 3.6 & 1.7 \\
\hline Sergestidae & 1.9 & 0.9 & - & - & - & - & - & - & - & - \\
\hline Pasiphaea multidentata & 1.9 & 0.9 & - & - & - & - & - & - & - & - \\
\hline Benthonic & 27.9 & 15.9 & 13.6 & 13.5 & 9.8 & 7.2 & 7.1 & 3.6 & 12.5 & 7.6 \\
\hline Aristeus antennatus & 18.3 & 8.2 & - & - & - & - & - & - & - & - \\
\hline Plesionika sp. & 4.8 & 2.6 & 4.5 & 2.7 & - & - & - & - & - & - \\
\hline Acanthephyra sp. & 1.0 & 0.4 & - & - & - & - & 4.0 & 2.1 & 7.1 & 3.4 \\
\hline Pontophilus norvegicus & 1.9 & 1.7 & 4.5 & 2.7 & 9.8 & 7.2 & 3.0 & 1.5 & 5.4 & 2.5 \\
\hline Alpheus glaber & 1.0 & 0.4 & 4.5 & 2.7 & - & - & - & - & - & - \\
\hline Unidentified & 6.7 & 2.6 & 9.1 & 5.4 & - & - & 1.0 & 0.5 & 3.6 & 1.7 \\
\hline Macrura & 29.8 & 15.1 & 18.2 & 8.1 & 2.4 & 1.8 & 1.0 & - & 1.8 & 0.8 \\
\hline Calocaris macandreae & 28.8 & 14.7 & 13.6 & 8.1 & 2.4 & 1.8 & - & - & - & - \\
\hline Polychelidae & 1.0 & 0.4 & - & - & - & - & 1.0 & 0.5 & 1.85 & 0.8 \\
\hline Anomura & - & - & - & - & 4.9 & 3.6 & 2.0 & 1.0 & - & - \\
\hline Pagurus alatus & - & - & - & - & 2.4 & 1.8 & - & - & - & - \\
\hline Munida tenuimana & - & - & - & - & 2.4 & 1.8 & 2.0 & 1.0 & - & - \\
\hline Brachyura & 1.0 & 0.4 & - & - & - & - & - & - & - & - \\
\hline Unidentified & 6.7 & 3.0 & - & - & 2.4 & 1.8 & - & - & - & - \\
\hline Euphausiacea & 1.0 & 0.4 & 13.6 & 8.1 & - & - & - & - & - & - \\
\hline Mysidacea & 17.3 & 15.6 & 4.5 & 2.7 & 14.6 & 10.8 & 13.1 & 6.7 & 16.1 & 7.6 \\
\hline Boreomysis arctica & 12.5 & 12.6 & 4.5 & 2.7 & 12.2 & 7.2 & 13.1 & 6.7 & 16.1 & 7.6 \\
\hline Parapseudomma sp. & 2.9 & 1.7 & - & - & 2.4 & 3.6 & - & - & - & - \\
\hline Unidentified & 1.9 & 1.3 & - & - & - & - & - & - & - & - \\
\hline Amphipoda & & & & & & & & & & \\
\hline Gammaridea & 2.9 & 6.5 & 9.1 & 8.1 & 9.8 & 7.2 & 9.1 & 4.6 & 5.4 & 2.4 \\
\hline Lysianassidae & - & - & 4.5 & 5.4 & 2.4 & 1.8 & - & - & - & - \\
\hline Harpinia sp. & - & - & - & - & - & - & 1.0 & 0.5 & 1.8 & 0.8 \\
\hline Leucothoe sp. & - & - & - & - & - & - & 1.0 & 0.5 & - & - \\
\hline Oedicerotidae & - & 0.4 & 4.5 & 2.7 & - & - & - & - & 1.8 & 0.8 \\
\hline Eusirus longipes & 1.0 & 0.9 & - & - & - & - & - & - & - & - \\
\hline Rhachotropis sp. & 1.0 & 2.6 & - & - & 4.9 & 3.6 & 4.0 & 2.1 & 1.8 & 0.8 \\
\hline Other Gammaridea & 1.0 & 1.3 & - & - & 2.4 & 1.8 & - & - & - & - \\
\hline Unidentified & 1.0 & 1.3 & - & - & - & - & 3.0 & 1.5 & - & - \\
\hline Isopoda & 7.7 & 7.8 & 4.5 & 2.7 & 19.5 & 14.5 & 10.1 & 5.1 & 5.4 & 6.1 \\
\hline Cirolana borealis & 7.7 & 5.2 & 4.5 & 2.7 & - & - & - & - & - & - \\
\hline Munnopsurus atlanticus & - & 2.2 & - & - & 17.1 & 12.7 & 6.1 & 3.1 & 1.8 & 0.8 \\
\hline Ilyarachna sp. & 1.0 & 0.4 & - & - & - & - & 2.0 & 1.0 & 3.6 & 2.5 \\
\hline Other Paraselloidea & - & - & - & - & 2.4 & 1.8 & 2.0 & 1.0 & 1.8 & 0.8 \\
\hline Tanaideacea & - & - & - & - & - & - & 4.0 & 2.6 & 1.8 & 1.7 \\
\hline Apseudomorpha & - & - & - & - & - & - & 1.0 & 0.5 & - & - \\
\hline Tanaiidomorpha & - & - & - & - & - & - & 4.0 & 2.1 & 1.8 & 1.7 \\
\hline Cumacea & 1.9 & 0.9 & - & - & - & - & 2.0 & 1.0 & 5.4 & 5.8 \\
\hline Epileucon longirostris & 1.9 & 0.9 & - & - & - & - & - & - & - & - \\
\hline Cyclaspis longicaudata & - & - & - & - & - & - & 1.0 & 0.5 & 1.8 & 0.8 \\
\hline Unidentified & - & - & - & - & - & - & 1.0 & 0.5 & 3.6 & 1.7 \\
\hline Ostracoda Platycopa & - & - & - & - & - & - & - & - & 5.4 & 3.3 \\
\hline Copepoda Harpacticoidea & - & - & - & - & - & - & 2.0 & 1.0 & 1.8 & 0.8 \\
\hline Unidentified & 1.9 & 0.9 & - & - & - & - & 4.9 & 1.0 & 2.0 & 2.5 \\
\hline
\end{tabular}


Table 1 (continued)

\begin{tabular}{|c|c|c|c|c|c|c|c|c|c|c|}
\hline \multirow{5}{*}{ No. of specimens: } & \multicolumn{6}{|c|}{ Polycheles typhlops } & \multicolumn{4}{|c|}{ Stereomastis sculpta } \\
\hline & \multicolumn{4}{|c|}{$550-750 \mathrm{~m}$} & \multirow{3}{*}{\multicolumn{2}{|c|}{$\begin{array}{c}1000-1200 \mathrm{~m} \\
\mathrm{Jul}-\mathrm{Oct} \\
41\end{array}$}} & \multirow{3}{*}{\multicolumn{2}{|c|}{$\begin{array}{c}1400-1900 \mathrm{~m} \\
\text { Jul-Oct } \\
99\end{array}$}} & \multirow{3}{*}{\multicolumn{2}{|c|}{$\begin{array}{c}2000-2300 \mathrm{~m} \\
\text { Jul } \\
56\end{array}$}} \\
\hline & \multirow{2}{*}{\multicolumn{2}{|c|}{$\begin{array}{c}\text { Mar-Jul } \\
104\end{array}$}} & \multirow{2}{*}{\multicolumn{2}{|c|}{$\begin{array}{l}\text { Oct } \\
22\end{array}$}} & & & & & & \\
\hline & & & & & & & & & & \\
\hline & $\% \mathrm{~F}$ & $\% \mathrm{~N}$ & $\% \mathrm{~F}$ & $\% N$ & $\% \mathrm{~F}$ & $\% \mathrm{~N}$ & $\% \mathrm{~F}$ & $\% \mathrm{~N}$ & $\% \mathrm{~F}$ & $\% \mathrm{~N}$ \\
\hline \multicolumn{11}{|l|}{ MOLLUSCA } \\
\hline Bivalvia & - & - & - & - & - & - & 2.0 & 1.0 & - & - \\
\hline Gastropoda & 3.8 & 1.8 & - & - & 19.5 & 12.7 & 60.6 & 35.0 & 39.3 & 20.2 \\
\hline Pteropoda & 1.9 & 0.9 & - & - & 19.5 & 12.7 & 60.6 & 34.5 & 39.3 & 20.2 \\
\hline Alvania sp. & 1.0 & 0.4 & - & - & - & - & - & - & - & - \\
\hline Benthonella tenella & 1.0 & 0.4 & - & - & - & - & 1.0 & 0.5 & - & - \\
\hline Cephalopoda & 4.8 & 2.2 & 4.5 & 2.7 & 7.3 & 5.4 & - & - & - & - \\
\hline Unidentified & - & - & - & - & - & - & 3.0 & 1.0 & - & - \\
\hline ECHINODERMATA & 3.8 & 1.7 & 27.3 & 16.2 & - & - & 6.1 & 3.1 & 10.7 & 5.0 \\
\hline Echinoidea & 1.0 & 0.4 & 27.3 & 16.2 & - & - & 1.0 & 0.5 & 1.8 & 0.8 \\
\hline Ophiuroidea & 2.9 & 1.3 & - & - & - & - & - & - & - & - \\
\hline Brisingella coronata & - & - & - & - & - & - & 5.1 & 2.6 & 8.9 & 4.2 \\
\hline OSTEICHTHYES & 46.2 & 22.6 & 54.5 & 32.4 & 26.8 & 18.2 & 33.3 & 17.9 & 37.5 & 19.3 \\
\hline Cyclothone sp. & - & - & - & - & - & - & 1.0 & 0.5 & - & - \\
\hline Myctophidae & 6.7 & 3.5 & 9.1 & 5.4 & - & - & 3.0 & 1.5 & 5.4 & 2.5 \\
\hline Macrouridae & 5.8 & 2.6 & 9.1 & 5.4 & - & -. & 2.0 & 1.0 & 7.1 & 3.4 \\
\hline Other osteichthyes & 33.7 & 16.5 & 36.4 & 21.6 & 26.8 & 18.2 & 29.3 & 14.9 & 28.6 & 13.4 \\
\hline Turridae eggs & - & - & - & - & 2.4 & 1.8 & - & - & - & - \\
\hline OTHER & 1.9 & 0.9 & 4.5 & 0.9 & 4.8 & 3.6 & 7.1 & 3.6 & 1.8 & 0.8 \\
\hline UNIDENTIFIED & 1.9 & 0.9 & - & 0.9 & - & - & 5.1 & 2.6 & 1.8 & 0.8 \\
\hline FORAMINIFERA & 10.6 & & 4.5 & & 36.6 & & 61.6 & & 46.4 & \\
\hline Benthic foraminifera & 6.8 & & 4.5 & & 4.8 & & 14.1 & & 7.2 & \\
\hline Globergerinae & 6.7 & & - & & 36.6 & & 61.65 & & 46.4 & \\
\hline Mud & - & & 4.5 & & 12.2 & & 22.2 & & 8.9 & \\
\hline Micromollusc remains & 1.0 & & 4.5 & & 4.9 & & 56.6 & & 46.4 & \\
\hline
\end{tabular}

pod communities (Cartes 1991). Both groups exhibited very similar diets (see below, 'Diversity and dietary overlap').

Numerical values were highest for pteropod shells, foraminiferans, and fish remains. In terms of abundance small calcified benthic ostracods were also important (Table 1).

Pteropod remains were the most important prey item in volume (Fig. 3). These shells bore attached bottom mud, small encrusting foraminiferans (Ammolagena sp.), minute sponges, and polyps (Stephanoscyphus spp.).

\section{Size differences in diet composition}

Polycheles typhlops. The following results are based on individuals from the upper middle slope, where the highest abundances of $P$. typhlops occur. The diet was quite similar in the 2 size classes larger than $20 \mathrm{~mm} \mathrm{CL}$ (Table 2; see also Table 4).

Immature Polycheles typhlops (smaller than $20 \mathrm{~mm}$ $\mathrm{CL}$ ) prey mainly on suprabenthic organisms, in par-
Fig. 3. Stereomastis sculpta. Proportion (by volume) of prey species by group in the diet by depth interval. Acan: Acanthephyra sp.; FOR: foraminiferans; PTER: pteropod remains; PLANT: plant matter. For explanation of all other abbreviations see Fig. 2
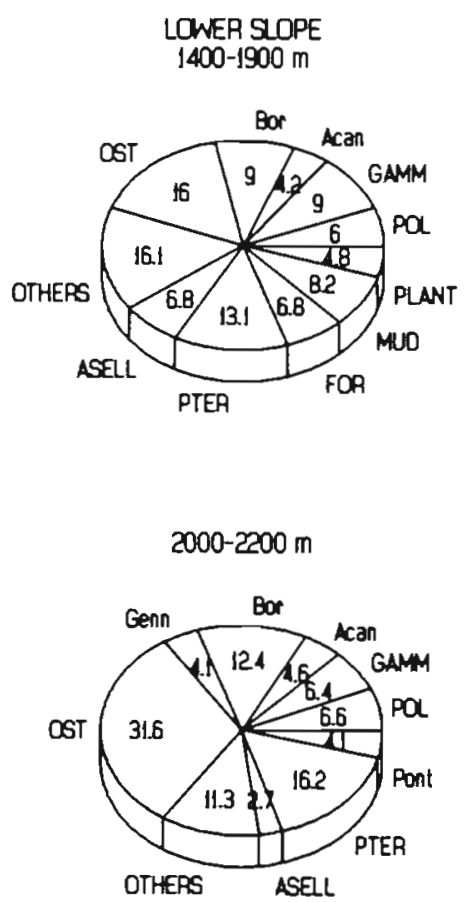
Table 2. Polycheles typhlops. Diet composition by size class in the depth range 550 to $750 \mathrm{~m}$

\begin{tabular}{|c|c|c|c|c|c|c|}
\hline \multirow[t]{2}{*}{ No. of specimens: } & \multicolumn{2}{|c|}{$\begin{array}{c}\mathrm{CL}>30 \mathrm{~mm} \\
66\end{array}$} & \multicolumn{2}{|c|}{$\begin{array}{c}30>\mathrm{CL}>20 \\
37\end{array}$} & \multicolumn{2}{|c|}{$\begin{array}{c}\mathrm{CL}<20 \mathrm{~mm} \\
24\end{array}$} \\
\hline & $\% \mathrm{~F}$ & $\% \mathrm{~N}$ & $\% \mathrm{~F}$ & $\% N$ & $\% F$ & $\% \mathrm{~N}$ \\
\hline POLYCHAETA & 1.5 & 0.9 & 5.4 & 2.9 & 4.2 & 2.1 \\
\hline Aphroditida & - & - & - & - & 4.2 & 2.1 \\
\hline Nephthys sp. & - & - & 2.7 & 1.4 & - & - \\
\hline Unidentified & 1.5 & 0.9 & 2.7 & 1.4 & - & - \\
\hline \multicolumn{7}{|l|}{ CRUSTACEA } \\
\hline \multicolumn{7}{|l|}{ Decapoda natantia } \\
\hline Bathypelagic & 3.0 & 1.8 & 5.4 & 2.8 & - & - \\
\hline Pasiphaea multidentata & 1.5 & 0.9 & 2.7 & 1.4 & - & - \\
\hline Sergestes arcticus & 1.5 & 0.9 & 2.7 & 1.4 & - & - \\
\hline Benthic & 27.3 & 16.1 & 29.7 & 15.7 & 8.4 & 4.2 \\
\hline Aristeus antennatus & 16.7 & 9.8 & 21.6 & 11.4 & - & - \\
\hline Plesionika (acanthonotus) & 6.1 & 3.6 & 2.7 & 1.4 & 4.2 & 2.1 \\
\hline Acanthephyra eximia & 1.5 & 0.9 & - & - & & \\
\hline Pontophilus norvegicus & 1.5 & 0.9 & 5.4 & 2.9 & 4.2 & 2.1 \\
\hline Alpheus glaber & 1.5 & 0.9 & - & - & - & - \\
\hline Unidentified & 4.5 & 2.7 & 5.4 & 2.9 & - & - \\
\hline Calocaris macandreae & 28.8 & 17.0 & 35.1 & 18.6 & 8.4 & 4.3 \\
\hline Polycheles typhlops & - & - & 2.7 & 1.4 & - & - \\
\hline Brachyura & 1.5 & 0.9 & - & - & - & - \\
\hline Unidentified & 6.1 & 3.6 & 8.1 & 4.3 & - & - \\
\hline Euphausiacea (Meganyctiphanes norvegica) & 1.5 & 0.9 & - & - & - & - \\
\hline Mysidacea & 15.2 & 8.9 & 21.6 & 11.5 & 45.8 & 36.1 \\
\hline Boreomysis arctica & 10.6 & 6.2 & 16.2 & 8.6 & 54.2 & 31.9 \\
\hline Paraseudommasp. & 1.5 & 0.9 & 5.4 & 2.9 & 4.2 & 2.1 \\
\hline Unidentified & 3.0 & 1.8 & - & - & 4.2 & 2.1 \\
\hline Amphipoda Gammaridea & 4.5 & 2.7 & 2.7 & 1.4 & 33.3 & 23.2 \\
\hline Oedicerotidae & - & - & - & - & 4.2 & 2.1 \\
\hline Eusirus logipes & - & - & 2.7 & 1.4 & 4.2 & 2.1 \\
\hline Rhachotropis sp. & 1.5 & 0.9 & - & - & 20.8 & 10.6 \\
\hline Other Gammaridea & 1.5 & 0.9 & - & - & 8.4 & 4.2 \\
\hline Unidentified & 1.5 & 0.9 & - & - & 8.4 & 4.2 \\
\hline Isopoda & 6.1 & 3.6 & 13.5 & 7.1 & 37.5 & 19.1 \\
\hline Cirolana borealis & 6.1 & 3.6 & 10.8 & 5.7 & 16.7 & 8.5 \\
\hline Munnopsurus atlanticus & - & - & - & - & 20.8 & 10.6 \\
\hline Ilyarachna sp. & - & - & 2.7 & 1.4 & - & - \\
\hline Cumacea (Epileucon longirostris) & 1.5 & 0.9 & 2.7 & 1.4 & - & - \\
\hline Unidentified & 1.5 & 0.9 & 2.7 & 1.4 & 4.2 & 2.1 \\
\hline \multicolumn{7}{|l|}{ MOLLUSCA } \\
\hline Gastropoda & 4.5 & 2.7 & 8.1 & 1.4 & - & - \\
\hline Pteropoda & 1.5 & 0.9 & 2.7 & 1.4 & - & - \\
\hline Alvania sp. & 1.5 & 0.9 & - & - & - & - \\
\hline Benthonella tenella & 1.5 & 0.9 & - & - & - & - \\
\hline Cephalopoda & 4.5 & 2.7 & 5.4 & 2.9 & - & - \\
\hline ECHINODERMATA & 4.5 & 2.7 & 2.7 & 1.4 & - & - \\
\hline Echinoidea & 1.5 & 0.9 & - & - & - & - \\
\hline Ophiuroidea & 3.0 & 1.8 & 2.7 & 14 & - & - \\
\hline OSTEICHTHYES & 48.5 & 28.6 & 40.5 & 21.5 & 16.7 & 8.5 \\
\hline Myctophidae & 6.1 & 3.6 & 5.4 & 2.9 & 4.2 & 2.1 \\
\hline Macrouridae & 6.1 & 3.6 & 5.4 & 2.9 & - & - \\
\hline Unidentified & 36.4 & 21.4 & 29.7 & 15.7 & 12.5 & 6.4 \\
\hline OTHERS & 3.0 & 1.8 & - & - & - & - \\
\hline UNIDENTIFIED & 1.5 & 0.9 & 2.7 & 1.4 & - & - \\
\hline FORAMINIFERA & 15.1 & & 5.4 & & 29.2 & \\
\hline Benthic foraminifera & 9.1 & & 5.4 & & 8.3 & \\
\hline Globigerinae & 9.1 & & 2.7 & & 20.7 & \\
\hline Mud & 1.5 & & - & & - & \\
\hline Micromollusc remains & 3.0 & & - & & - & \\
\hline
\end{tabular}


Table 3. Stereomysis sculpta. Diet composition by size class in the depth range 1400 to $2300 \mathrm{~m}$

\begin{tabular}{|c|c|c|c|c|c|c|}
\hline \multirow[t]{2}{*}{ No. of specimens: } & \multicolumn{2}{|c|}{$\begin{array}{c}\mathrm{Cl}>25 \mathrm{~mm} \\
40\end{array}$} & \multicolumn{2}{|c|}{$25>\underset{72}{C L}>16$} & \multicolumn{2}{|c|}{$\begin{array}{c}\mathrm{CL}<16 \mathrm{~mm} \\
19\end{array}$} \\
\hline & $\% \mathrm{~F}$ & $\% \mathrm{~N}$ & $\% F$ & $\% \mathrm{~N}$ & $\% F$ & $\% \mathrm{~N}$ \\
\hline POLYCHAETA & - & - & 12.2 & 6.4 & 26.3 & 13.1 \\
\hline Aphroditida & - & - & 6.8 & 3.3 & 21.0 & 10.5 \\
\hline Glycera sp. & - & - & 1.4 & 0.7 & - & - \\
\hline Unidentified & - & - & 5.6 & 2.7 & 5.3 & 2.6 \\
\hline NEMATODA & 2.5 & 1.1 & 1.4 & 0.7 & 5.3 & 2.6 \\
\hline \multicolumn{7}{|l|}{ CRUSTACEA } \\
\hline \multicolumn{7}{|l|}{ Decapoda } \\
\hline Gennadas elegans & 2.5 & 1.1 & 2.8 & 1.3 & - & - \\
\hline Acanthephyra sp. & 7.5 & 3.4 & 4.2 & 2.0 & - & - \\
\hline Pontophilus norvegicus & 2.5 & 1.1 & 5.6 & 2.7 & 5.3 & 2.6 \\
\hline Unidentified natantia & - & - & 2.8 & 1.3 & - & - \\
\hline Stereomastis sculpta & 2.5 & 1.1 & - & - & - & - \\
\hline Munida tenuimana & 2.5 & 1.1 & 1.4 & 0.7 & - & - \\
\hline Mysidacea & 7.5 & 3.4 & 16.2 & 7.9 & 31.6 & 13.2 \\
\hline Boreomysis arctica & 7.5 & 3.4 & 16.2 & 7.9 & 31.6 & 13.2 \\
\hline Amphipoda Gammaridea & 7.5 & 3.4 & 6.9 & 3.4 & 21.0 & 10.5 \\
\hline Harpinia sp. & - & - & 1.4 & 0.7 & - & - \\
\hline Leucothoe incisa & - & - & - & - & 5.3 & 2.6 \\
\hline Oedicerotidae & 2.5 & 1.1 & - & - & - & - \\
\hline Rhachotropis sp. & 2.5 & 1.1 & 4.2 & 2.0 & -. & - \\
\hline Unidentified & 2.5 & 1.1 & 1.4 & 0.7 & 15.8 & 7.9 \\
\hline Isopoda & 12.5 & 5.6 & 8.3 & 4.7 & 26.3 & 13.2 \\
\hline Munnopsurus atlanticus & 2.5 & 1.1 & 5.6 & 2.7 & 10.5 & 5.3 \\
\hline llyarachna sp. & 7.5 & 3.4 & 1.4 & 0.7 & 10.5 & 5.3 \\
\hline Other Paraselloidea & 2.5 & 1.1 & 2.8 & 1.3 & 5.3 & 2.6 \\
\hline Tanaidacea & 10.0 & 4.5 & 4.2 & 2.0 & - & - \\
\hline Apseudomorpha & - & - & 1.4 & 0.7 & - & - \\
\hline Tanaiidomorpha & 10.0 & 4.5 & 2.8 & 1.3 & - & - \\
\hline Cumacea & 7.5 & 4.5 & - & - & 5.3 & 2.6 \\
\hline Cyclaspis longicaudata & 5.0 & 2.3 & - & - & - & - \\
\hline Unidentified & 2.5 & 1.1 & - & - & 5.3 & 2.6 \\
\hline Ostracoda & 10.0 & 16.9 & - & - & - & - \\
\hline Myodocopa & - & - & - & - & 5.3 & 2.6 \\
\hline Ostracoda Platycopa & 10.0 & 16.9 & - & - & - & - \\
\hline Copepoda Harpacticoida & 2.5 & 1.1 & 2.8 & 1.3 & - & - \\
\hline Unidentified & - & - & 4.2 & 2.0 & - & - \\
\hline \multicolumn{7}{|l|}{ MOLLUSCA } \\
\hline Bivalvia & - & - & 2.8 & 1.3 & - & - \\
\hline Gastropoda & 57.5 & 31.5 & 51.4 & 30.6 & 42.1 & 18.4 \\
\hline Pteropoda & 57.5 & 31.5 & 51.4 & 29.1 & 42.1 & 18.4 \\
\hline Benthonella tenella & - & - & 1.4 & 0.7 & - & - \\
\hline Unidentified & 2.5 & 1.1 & 1.4 & 0.7 & - & - \\
\hline ECHINODERMATA & 2.5 & 1.1 & 11.1 & 5.3 & 5.3 & 2.6 \\
\hline Echinoidea & - & - & 2.8 & 1.3 & - & - \\
\hline Brisingella coronata & 2.5 & 1.1 & 8.3 & 4.0 & 5.3 & 2.6 \\
\hline OSTEICHTHYES & 35.0 & 15.7 & 38.9 & 19.3 & 21.0 & 10.5 \\
\hline Myctophidae & 2.5 & 1.1 & 5.6 & 2.7 & - & - \\
\hline Macrouridae & - & - & 5.6 & 2.7 & - & - \\
\hline Unidentified & 32.5 & 14.6 & 29.2 & 13.9 & 21.0 & 10.5 \\
\hline OTHERS (Plant debris) & 7.5 & 3.4 & 6.9 & 3.3 & 5.3 & 2.6 \\
\hline UNIDENTIFIED & - & - & 8.3 & 4.0 & 5.3 & 2.6 \\
\hline FORAMINIFERA & 82.5 & & 65.3 & & 38.9 & \\
\hline Benthic foraminifera & 15.0 & & 8.4 & & 15.9 & \\
\hline Globigerinae & 82.5 & & 61.1 & & 31.6 & \\
\hline Mud & 30.0 & & 18.1 & & 10.6 & \\
\hline Mollusc remains & 60.0 & & 52.8 & & 36.8 & \\
\hline
\end{tabular}


ticular mysids (Boreomysis arctica, Parapseudomma calloplura), gammarid amphipods (Rhachotropis grimaldii, Eusirus longipes, Niccipe tumida), and isopods (Munnopsurus atlanticus, Cirolana borealis). In all, suprabenthic crustaceans made up $82.7 \%$ of the total diet. Remains attributable to scavenging activity were of rather minor importance $(7 \%)$.

Dietary overlap between the 2 largest size classes and the smallest size class was very low (see Table 6).

Stereomastis sculpta. The diets of the 2 largest size classes were dominated by pteropod and fish remains (Table 3), which accounted for nearly $50 \%$ of the diet. In immature individuals the proportion of these 2 prey items in the diet dropped to $29 \%$. The share of suprabenthic crustaceans (45\%) grew in importance in the diet of the smallest size class, whereas the contribution of this group of prey was much lower in the medium and large size classes.

The mysid Boreomysis arctica and the asellid isopods Munnopsurus atlanticus, Ilyarachna sp., and Janirella sp. were particularly important in the diet of the smallest individuals.

\section{Feeding activity}

The proportion of empty stomachs was very high in both species (Table 4 ). In Polycheles typhlops $67.8 \%$ of all individual stomachs either were empty or contained few food remains. The proportion was even higher $(74.3 \%)$ in Stereomastis sculpta.

No significant differences were found in the degree of foregut fullness at different times of the day in either species ( $p \leq 0.05)$, though perhaps they may be

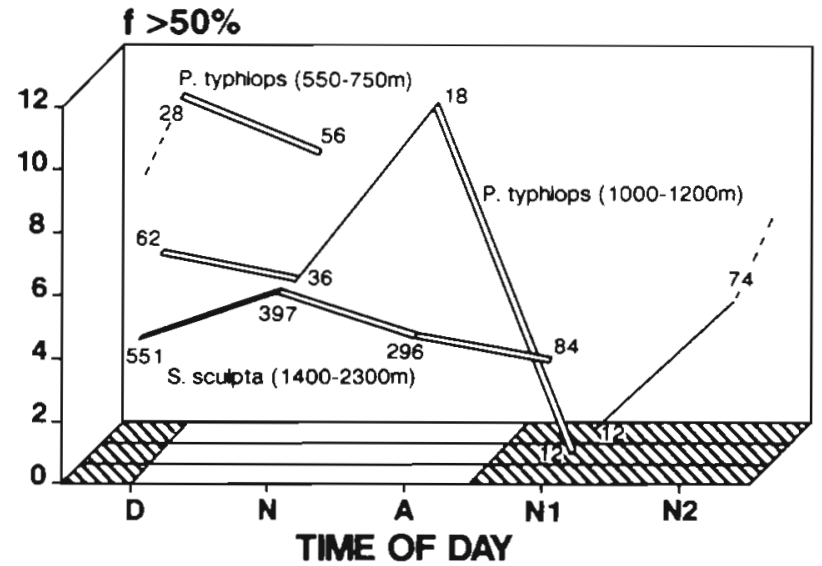

Fig. 4. Polycheles typhlops and Stereomastis sculpta. Percentage of full stomachs by depth according to time of day. Number of specimens appears next to each data point. D: ddybreak; N: noon; A: afternoon; N1, N2: nighttime

important in view of the low feeding intensity recorded in these 2 species (Fig. 4). Very few differences in the type of prey ingested were also recorded at the periods considered.

\section{Diversity and dietary overlap}

Trophic diversity in Stereomastis sculpta increased with depth (Table 4). This trend is not evident for Polycheles typhlops. There seem to be seasonal differences in the latter species.

Diversity in Polycheles typhlops was higher in the larger size classes (Table 5) than in individuals smaller

Table 4. Polycheles typhlops and Stereomastis sculpta. Trophic diversity and feeding activity by depth interval

\begin{tabular}{|lccccc|}
\hline & \multicolumn{3}{c|}{ Polycheles typhlops } & \multicolumn{2}{c|}{$\begin{array}{c}\text { Stereomastis sculpta } \\
\end{array}$} \\
& $\begin{array}{c}550-750 \mathrm{~m} \\
\text { Mar-Jul }\end{array}$ & $\begin{array}{c}550-750 \mathrm{~m} \\
\text { Oct }\end{array}$ & $\begin{array}{c}1000-1200 \mathrm{~m} \\
\text { Jul-Oct }\end{array}$ & $\begin{array}{c}1400-1900 \mathrm{~m} \\
\text { Jul-Oct }\end{array}$ & Jul \\
\hline No. of specimens & 191 & 58 & 139 & 727 & 412 \\
$f<20 \%$ & $56.3 \%$ & $52.6 \%$ & $76.9 \%$ & $78.2 \%$ & $79.9 \%$ \\
$f \geq 50 \%$ & $8.9 \%$ & $8.6 \%$ & $2.9 \%$ & $2.2 \%$ & $3.4 \%$ \\
Diversity $\left(H^{\prime}\right)$ & 4.307 & 3.668 & 3.561 & 3.754 & 4.117 \\
\hline
\end{tabular}

Table 5. Polycheles typhlops and Stereomastis sculpta. Trophic diversity and feeding activity by size class

\begin{tabular}{lcccccc}
\hline & \multicolumn{3}{c}{ Polycheles typhlops } & \multicolumn{2}{c}{ Stereomastis sculpta } \\
& CL $>30 \mathrm{~mm}$ & $30>\mathrm{CL}>20$ & $\mathrm{CL}<19 \mathrm{~mm}$ & $\mathrm{CL}>25 \mathrm{~mm}$ & $25>\mathrm{CL}>18$ & $\mathrm{CL}<17 \mathrm{~mm}$ \\
\hline No. of specimens & 148 & 115 & 74 & 227 & 841 \\
$f<20 \%$ & $69.7 \%$ & $72.6 \%$ & $62.4 \%$ & $75.3 \%$ & $79.9 \%$ & $80.6 \%$ \\
$f \geq 50 \%$ & $5.1 \%$ & $0.9 \%$ & $13.1 \%$ & $4.0 \%$ & $2.0 \%$ & $5.6 \%$ \\
Diversity $\left(H^{\prime}\right)$ & 4.093 & 3.980 & 3.668 & 3.438 & 3.966 & 3.671 \\
\hline
\end{tabular}


Table 6. Polycheles typhlops (Pt) and Stereomastis sculpta (Ss). Dietary overlap between the different groups established and between the different size classes in samples. UMS: upper middle slope; LMS: lower middle slope; LS: lower slope

\begin{tabular}{|c|c|c|c|c|c|c|}
\hline $\begin{array}{l}\text { Polycheles typhlops, } \\
\text { Stereomastis sculpta }\end{array}$ & $\begin{array}{c}\text { Pt (UMS) } \\
550-750 \mathrm{~m} \\
\text { Oct }\end{array}$ & & $\begin{array}{c}\text { Pt (LMS) } \\
1000-1200 \mathrm{~m} \\
\text { Jul-Oct }\end{array}$ & \multicolumn{2}{|c|}{$\begin{array}{c}\text { Ss (LS) } \\
1400-1900 \mathrm{~m} \\
\text { Jul-Oct }\end{array}$} & $\begin{array}{c}\text { Ss (LS) } \\
2000-2300 \mathrm{~m} \\
\text { Jul }\end{array}$ \\
\hline UMS (550-750 m, Jul) & 48.146 & & 41.273 & & & 987 \\
\hline UMS $(550-750 \mathrm{~m}$, Oct $)$ & - & & 32.108 & & & .593 \\
\hline LMS $(1000-1200 \mathrm{~m})$ & - & & - & & & 252 \\
\hline $\operatorname{LS}(1400-1900 \mathrm{~m})$ & - & & - & & & .538 \\
\hline Polycheles typhlops & $30<\mathrm{CL}<20$ & $\mathrm{CL}>20 \mathrm{~mm}$ & Stere & culpta & $25>\mathrm{CL}>17$ & $\mathrm{CL}<17 \mathrm{~mm}$ \\
\hline $\mathrm{CL}>30 \mathrm{~mm}$ & 77.857 & 31.858 & $\mathrm{CL}>$ & & 65.340 & 46.186 \\
\hline $30 \mathrm{~mm}>$ CL $20 \mathrm{~mm}$ & - & 35.593 & $25 \mathrm{~mm}$ & $7 \mathrm{~mm}$ & - & 59.306 \\
\hline
\end{tabular}

than $20 \mathrm{~mm} \mathrm{CL}$. In contrast, the largest Stereomastis sculpta size class did not show the highest diversity values. The maximum value of $H^{\prime}$ was shown by individuals in the medium size class (Table 5).

The overlap index values for the sample groups were generally low (Table 6). The maximum value occurred between samples of Stereomastis sculpta and samples of Polycheles typhlops from the lower middle slope $(1000$ to $1200 \mathrm{~m})$. The minimum value $(27.623)$ between species was between the diets in the most distant bathymetric zones.

The maximum overlap value between size classes within each species was between the medium and large size classes (Table 6). The minimum overlap value was between the extreme size classes (the smallest and the largest size classes) in both cases.

\section{DISCUSSION}

Polycheles typhlops and Stereomastis sculpta have similar morphologies and sizes. In addition, the depth areas in which each species is most abundant are distinctly separate (Abelló \& Cartes in press). These 2 species appear to occupy a similar niche, encompassing the uppermost level of the bottom sediment and the water layer immediately adjacent to the sea bed, judging from the predominance of prey items from these layers in their diets. Planktonic prey (hyperiids, siphonophores, chaetognaths, calanids, etc.) were almost completely absent. Such organisms are present in the diet of nektobenthic species like Aristeus antennatus (Cartes \& Sardà 1989), which are abundant in the same areas as polychelid lobsters. These prey groups are also present in samples collected with suprabenthic sleds equipped with automatic opening/closing systems, particularly in the upper subsample (50 to $100 \mathrm{~cm}$ from the bottom)
(Sorbe 1983, authors' unpubl. data).

The proportion of polychaetes and other endobenthic prey (other than Calocaris macandreae) was small, especially in Polycheles typhlops. Aphroditids and Nephthys sp. were the predominant polychaetes. These 2 groups are regarded as slow-moving predators on the surface of the sea bed (Fauchauld \& Jumars 1979). In contrast, glycerids were absent from the diets. Glycerids include species that dwell permanently in burrows, like Glycera rouxii, a predominant species in the bathyal macrobenthic mud assemblages in the Catalan Sea (Reyss 1971). Glycerids are prevalent in the diet of Aristeus antennatus, a species well equipped to root in the substrate in search of prey (Cartes \& Sardà 1989, Cartes 1991).

The gammarid amphipods identified were epibenthic and highly mobile, like the eusirids (Eusirus longipes, Rhachotropis sp.), regarded as active predators of calanids and other crustaceans, and the Lysianassids, generally regarded as scavengers (Lagardère 1976, Slattery \& Oliver 1986). In contrast, endobenthic species like Harpinia pectinata and Urot'roe corsica (Enequist 1949), common prey of $A$. antennatus, were almost absent from the diet.

Based on a few specimens of Stereomastis sculpta, Lagardère (1977) suggested that its diet was similar to that of Polycheles typhlops. In our results the maximum interspecific overlap value was obtained for the samples from the closest depth strata. This value, 54.144, was relatively high (Schoener 1974), indicating that the trophic habits of the 2 species in their areas of distribution overlap are similar. Habitat separation is important in preventing species competitive interactions (Schoener 1974, Rex 1977). This could be the case for $P$. typhlops and S. sculpta. Thus, only the deepest $P$. typhlops sample was associated with $S$. sculpta. In fact both species based their diets in similar prey groups with variation in prey species due to changes in ben- 
thic communities with depth (Pérès 1985, Cartes 1991 Stefanescu 1991).

Scavenging activity plays an important role in the feeding of both species, and both can be classified as active scavengers according to the classification of Lagardère (1976). The presence of large decapod crustaceans, fishes, and cephalopods remains can be ascribed to such scavenging activity. In all cases parts of these prey items were present, providing a means of gauging the size of the whole animal. Based on the estimated sizes, active hunting seems unlikely, particularly in view of the relatively unaggressive nature of Polycheles typhlops (Santucci 1932). The fish remains were chiefly scales, some vertebrae and/or spines, and in a very few instances only, small otoliths of Myctophidae or the nearly whole axial skeletons of small fish, probably the result of active predation.

The contribution of scavenging activity to the diet of adult Polycheles typhlops on the upper middle slope was nearly $40 \%$; the impact of this trophic strategy was much smaller in the diet of small individuals. This type of activity may have been stimulated by commercial trawier operations carried out in the region at depths down to between 700 and $800 \mathrm{~m}$, either directly or through discarding of non-commercial species. The importance of scavenging activity in the diet of $P$. typhlops on the lower middle slope decreased. In contrast, it was replaced by a new strategy, namely, the ingestion of pelagic matter from the sediment (pteropod remains and foraminiferans). Pteropod remains were abundant in the biogenic sediments of the lower slope in the Catalan Sea, while in less deeper zones the terrigenous sediments were dominant (Emelyanov 1977, Fredj \& Laubier 1985, Cartes 1991).

Scavenging behaviour made only a small contribution to the diet of Stereomastis sculpta, similar to Polycheles typhlops in the lower middle slope. It was complemented by the ingestion of pteropod remains, mud, plant debris, and/or foraminiferans.

Epibenthic prey were important in both species, above all in immature individuals. The presence of euphausiids (mainly Meganyctiphanes norvegica) in the samples taken in the upper middle slope in autumn was notable. This group is also present in the diets of all the other decapods inhabiting the middle slope at this same season (Cartes 1991). On the lower middle slope, the share of epibenthic prey and peracarid crustaceans became more important, accounting for over $40 \%$ of the diet.

The lower density of Polycheles typhlops on the lower middle slope as compared to the upper middle slope coincided with greatly reduced densities of some of this species' preferred prey items (Calocaris macandreae, Cirolana borealis and Meganyctiphanes norvegica), which were abundant in the upper middle slope (Pérès 1958, Carpine 1970, Reyss 1971, Cartes 1991) and with a different feeding strategy. These are likely factors contributing to the progressive decrease in the population of this species with depth.

On the middle slope both adult and immature individuals of Polycheles typhlops are abundant (Abello \& Cartes in press). However, only small P. typhlops were collected on the lower slope (between 1400 and $1900 \mathrm{~m}$ ). The diet of these small individuals was dominated largely by peracarid crustaceans, still abundant at those depths (Pérès 1985). Adults may not be able to $d$ well at those depths since their main prey items are unavailable. This appears to occur concomitantly with a decrease in the size of the macrobenthic fauna comprising the basic diet of polychelids at greater depths (Jumars \& Gallagher 1982, Pérès 1985, Sokolova 1990). The diet of small individuals on the lower slope was similar to that described for immature P. typhlops on the upper middle slope.

The presence of Stereomastis sculpta marks the lower limit of the distribution range of Polycheles typhlops. S. sculpta exhibits quite similar trophic preferences, and seems to be better adapted to environments with lower prey densities (Jumars \& Gallagher 1982, Rowe 1983, Thiel 1983, Pérès 1985) or with less nutritional food resources (pteropod remains or foraminiferans). The tendency of species that inhabit relatively unproductive (oligotrophic) zones like deep-sea bottoms to be more omnivorous and microphagous has been reported for a variety of invertebrate groups (Carey 1972, Sokolova 1972) and appears to be part of a general pattern of trophic adaptation to conditions in which resources are scarce. Thus, S. sculpta appears to match the definition of cropper species put forward by Dayton \& Hessler (1972) for deep-water predators. These results are in agreement with those reported for other deep-sea polychelids (Wenner 1979, Gore 1984).

Ontogenetic changes in diet were clearly seen in both species. On the whole, small individuals based their diet on epibenthic peracarid crustaceans, whereas scavenging activity and ingestion of inert remains from the bottom sediment became more important in the diets of medium and Iarge individuals. Santucci (1932), basing his study on large specimens, concluded that Polycheles typhlops was almost exclusively a scavenger. Lagardère (1977) in turn reported that the diet of $P$. typhlops was based on euphausiids and mysids. These findings partially agree with the results reported herein for sizes smaller than $30 \mathrm{~mm}$ CL. However, in the present samples euphausiids were important only seasonally.

The results for the diet of Stereomastis sculpta were to a certain extent parallel to those for Polycheles typhlops on the basis of size class. Thus, while the diet of adults was based on slow-moving or even sedentary 
prey items (mud, dead matter, etc.) juveniles were much more specialized, active predators of small prey like peracarids. Medium-sized individuals exploited both types of food resource, hence their diet was more diversified. However, for all the size classes combined as well as for each size class separately, S. sculpta tended to exploit less mobile prey items (polychaetes, benthic ostracods, etc.) more than $P$. typhlops did. The behaviour of this latter species, and especially that of immature individuals, was more consistent with the behaviour of a more active predator. Comparing the diets of the smallest size classes of the 2 species indicates that small $S$. sculpta are better adapted to exploit inert remains (pteropods), while small $P$. typhlops specialized nearly exclusively in the capture of live prey.

Feeding intensity was quite low in both species, particularly since the digestion rate must be relatively slow, given the low constant bottom temperature of $12.7^{\circ} \mathrm{C}$ in the bathyal Mediterranean regions. This was more pronounced in Stereomastis sculpta, despite the possible longer digestion times for calcareous remains (pteropods, foraminiferans) (Joll 1982) in the foregut of this species. The high proportion of empty stomachs has been reported previously by Wenner (1979) and Gore (1984) and would seem to be a general pattern in the Polychelidae as an adaptation to oligotrophic deepsea areas.

Lagardère (1977) reported predacious activity only during the daytime in Polycheles typhlops, justified by the presence of mesopelagic species that carry out vertical migrations and dwell close to the bottom during daytime hours. Our results do not show significant differences in daily feeding rhythm, probably due to the small influence of mesopelagic species in the diet.

In summary, the trophic habits of the 2 polychelid species in the Western Mediterranean were rather similar. The differences recorded seem to be more consistent with depth-related changes in the composition of available prey resources and faunal communities than to changes in the position of the two species in the food web. Moreover, various indicators suggest that the deeper-dwelling species, Stereomastis sculpta, is better adapted to a environment poorer in resources. A possible competitive trophic interaction may also account for the separate depth distributions of the 2 species, as postulated by Rex (1977). Such trophic interactions may be especially important on the deepsea bottoms of the Mediterranean Sea, where environmental parameters like temperature and salinity are constant.

Acknowledgements. The authors express their appreciation to all the members of the deep-sea research programs 'Batimar', 'A.bismar', 'Pont 89' and 'Pont 90' for their collaboration, especially to Drs F. Sardà and D. Lloris and Mr J.
Rucabado. Other colleagues helped us in the identification of different prey species. We are very grateful to Drs J. C. Sorbe and J. P. Lagardere for their help in the identification of different species, especially peracarid crustaceans, and to L. Dantart and R. Villanueva for their identification of the mollusc species. Thanks are due to R. Sacks who prepared the English translation of this paper and to $J$ M. Anguita for his technical assistance in preparing the figures.

\section{LITERATURE CITED}

Abelló, P., Cartes, J. E. (in press). Population characteristics of the deep-sea lobsters Polycheles typhlops and Stereomastis sculpta (Decapoda: Polychelidae) in a bathyal mud community of the Mediterranean Sea. Mar. Biol.

Abelló, P., Valladares, F. J. (1988). Bathyal decapod crustaceans of the Catalan Sea (Northwestern Mediterranean). Mésogée 48: 97-102

Abelló, P., Valladares, F. J., Castellón, A. (1988). Analysis of the structure of decapod crustacean assemblages off Catalan coast (North-West Mediterranean). Mar. Biol. 98: $39-49$

Amezaga, R. (1988). Análisis de los contenidos estomacales en peces. Revisión bibliográfica de los objetivos y la metodología. Inf. Tec. Inst. Esp. Oceanogr. 63: 1-74

Carey, A. G. (1972). Food sources of sublittoral, bathyal and abyssal asteroids in the northeast Pacific Ocean. Ophelia 10: $35-47$

Carpine, C. (1970). Ecologie de l'étage bathyal dans la Méditerranée occidentale. Mém. Inst. océanogr. Monaco 2: $1-146$

Cartes, J. E. (1991). Análisis de las comunidades y estructura trófica de los crustáceos decápodos batiales del Mar Catalán. Tesis doctoral, Universidad Politécnica de Catalunya

Cartes, J. E., Abelló, P. (1990). Comparative size distribution and feeding ecology of Polycheles typhlops and Stereomastis sculpta (Decapoda, Polychelidae) in the Mediterranean bathyal mud assemblage. Rapp. Comm. int. Mer Médit. 32 (1): 35

Cartes, J. E., Sardà, F. (1989). Feeding ecology of the deepwater aristeid crustacean Aristeus antennatus. Mar. Ecol. Prog. Ser. 54: 229-238

Cartes, J E., Sardà, F., Abelló, P. (in press). Decapod crustaceans collected in deep samplings (between 1000 and $2200 \mathrm{~m}$ ) in the Catalan area (Northwestern Mediterranean). Bios (Thessaloniki)

Dayton, P. K., Hessler, R. R. (1972). Role of biological disturbance in maintaining diversity in the deep sea. Deep Sea Res. $19: 199-208$

Emelyanov, E. M. (1972). Principal types of recent bottom sediments in the Mediterranean Sea : their mineralogy and geochemestry. In: Stanley, D. J. (ed.) The Mediterranean Sea: a natural sedimentation laboratory. Dowden, Hutchinson and Ross, Inc., Stroudsburg, p. 355-386

Enequist, P. (1949). Studies on the soft-bottom amphipods of the Skagerrak. Zool. Bidr. Uppsala 28: 297-492

Fauchald, K., Jumars, P. A. (1979). The diet of worms. A study of polychaete feeding guilds. Oceanogr. mar Biol. Ann. Rev. 17: 193-284

Fredj, G., Laubier, L. (1985). The deep Mediterranean benthos. In: Moraitou-Apostolopoulou, M., Kiortsis, V (eds.) Mediterranean marine ecosystems. Plenum Press, New York, p. 109-146 
Gore, R. H. (1984). Notes on abyssal lobsters, genus Willemoesia (Palinura, Polychelidae) collected by USNS 'Bartlett' from the Venezuela Basin, Caribbean Sea. Proc. Acad. natn. Sci. Philadelphia $136: 1-11$

Hopkins, T S. (1985). Physics of the sea. In: Margalef, R. (ed.) Key environments: Western Mediterranean. Pergamon Press, New York, p. 100-125

Hyslop, E. J. (1980). Stomach contents analysis. A review of methods and their application. J. Fish. Biol. 17: 411-429

Joll, L. M. (1982). Foregut evacuation of four foods by western rock lobster, Panulirus cygnus, in aquaria. Aust. J. mar. Freshwat. Res. 33: 939-943

Jumars, P. A., Gallagher, E. D. (1982). Deep-sea community structure: three plays on the Benthic Proscenium. In: Ernst, W. G., Morin, V. G. (eds.) The environment of the deep-sea. Prentice-Hall, Englewood Cliffs, New Jersey, p. 217-254

Kensley, B. F. (1968). Deep sea decapod Crustacea from west of Cape Point, South Africa. Ann. S. Afr. Mus. 50 (12): $283-323$

Lagardère, J P. (1976). Recherches sur la distribution verticale et sur l'alimentation des Crustacés Décapodes de la Pente continentale de I'Atlantique Nord-oriental. Thèse Univ. Aix-Marseille II, Arch. orig. C. N. R. S., 12. 237

Lagardère, J. P. (1977). Recherches sur la distribution verticale et sur l'alimentation des crustaces decapodes benthiques de la Pente Continentale du Golfe de Gascogne. Analyse des groupements carcinologiques. Bull. Cent. Etud. Rech. sci. Biarritz, 11 (4): 367-440

Mauchline, J., Gordon, J. D. M. (1985). Trophic diversity in deep-sea fish. J. Fish Biol. 26: 527-535

Merret, N., Marshall, N. B. (1981). Observations of the ecology of deep-sea bottom-living fishes collected off northwest Africa $\left(08^{\circ}-27^{\circ} \mathrm{N}\right)$. Prog. Oceanogr. 9: 185-244

Pérès, J. M. (1958). Remarques générales sur un ensemble de quinze plongées effectuées avec le bathyscaphe F. N. R. S. III. Annls Inst. océanogr. Monaco 35: 259-285

Pérès, J. M. (1985). History of the Mediterranean biota and the colonization of the depths. In: Margalef, R. (ed.) Key environments: Western Mediterranean. Pergamon Press, New York, p. 198-232

Rex, M. A. (1977). Zonation in deep-sea gastropods: the importance of biological interactions to rates of zonation. In: Keegan, B. F., Ceidigh, P. O., Boaden, P. J. S. (eds.) Biology of benthic organisms. Pergamon Press, New York, p. $521-530$

Reyss, D. (1971). Les canyons sous-marins de la mer Catalane: le rech du Cap et le rech Lacaze-Duthiers. III. Les

This aticle was submitted to the editor peuplements de macrofaune benthique. Vie Milieu 22 : $529-613$

Rowe, G. T. (1983). Biomass and production of the Deep-sea Macrobenthos. In: Rowe, G. T. (ed.) Deep-sea biology, Vol. 8, The sea. John Wiley \& Sons, New York, p. 97-121

Saint Laurent, M. (1985). Remarques sur la distribution des crustacés décapodes. In: Laubier, L., Monniot, C. (eds.) Peuplements profonds du golfe de Gascogne. IFREMER, Brest, p. $469-478$

Santucci, R. (1932). Biologia del fondo a 'scampi' nel mare ligure. I. Polycheles typholps Heller. Memorie R. Com. talassogr. ital. 199: 1-48

Sardà, F., Valladares, F. J. (1990). Gastic evacuation of different foods by Nephrops norvegicus (Crustacea: Decapoda) and estimation of soft tissues ingested, maximum food intake and cannibalism in captivity. Mar Biol. 104: 25-30

Schoener, T. W. (1974). Resource partitioning in ecological communities. Science 185: 27-39

Shannon, C. E., Weaver, W. (1963). The mathematical theory of communication. Urbana Press, Univ. Illinois, Urbana

Slattery, P. N., Oliver, J. S., (1986). Scavenging and other feeding habits of lysianassid amphipods (Orchomene spp.) from McMurdo Sound, Antarctica. Polar Biol. 6: 171-177

Sokolova, M. N. (1972). Trophic structure of deep-sea macrobenthos. Mar. Biol. 16: 1-12

Sokolova, M. N. (1990). On the size of the deep-sea macrobenthic invertebrates. Prog. Oceanogr. 24: 251-252

Sorbe, J. C. (1983). Description d'un traineau destiné à l'échantillonnage quantitatif étagé de la faune suprabenthique néritique. Ann. Inst. océanogr. Paris 59 (2): 117-126

Stefanescu, C. (1991). Comunidades ictiológicas demersales del Mar Catalán (Mediterraneo noroccidental) por debajo de los $1000 \mathrm{~m}$ de profundidad. Tesis doctoral, Universidad de Barcelona

Swynnerton, G. H., Worthington, E. B. (1940). Note on the food of fish in Haweswater (Westmoreland). J. Anim. Ecol. 9: $183-187$

Thiel, H. (1983). Meiobenthos and nanobenthos of the deep sea. In: Rowe, G. T. (ed.) Deep-sea biology, Vol. 8, The sea. John Wiley \& Sons, New York, p. 167-230

Wenner, E. L. (1979). Some aspects of the biology of deep-sea lobsters of the family Polychelidae (Crustacea, Decapoda) from the western North Atlantic. Fish. Bull. U.S. 77 (2): $435-444$

Wenner, E. L., Boesch, D. F. (1979). Distribution patterns of epibenthic decapod Crustacea along the shelf-slope coenocline, middle Atlantic Bight, USA. Bull. Biol. Soc. Wash. 3: $106-133$

Manuscript first received: December 5, 1991

Revised version accepted: May 12, 1992 